\title{
A Note on the Singh Six-order Variant of Newton's Method
}

\author{
Andrzej Marciniak ${ }^{1,2}$, Marek Wolf ${ }^{3}$ \\ 1* Poznan University of Technology, Institute of Computing Science \\ Piotrowo 2, 60-965 Poznań, Poland \\ E-mail: Andrzej.Marciniak@put.poznan.pl \\ ${ }^{2}$ Higher Vocational State School in Kalisz, Department of Computer Science \\ Poznanska 201-205, 62-800, Kalisz, Poland \\ ${ }^{3}$ Cardinal Stefan Wyszynski University \\ Faculty of Mathematics and Natural Sciences, College of Sciences \\ ul. Wóycickiego 1/3, Auditorium Maximum, (room 113) \\ PL-01-938 Warsaw, Poland \\ E-mail:m.wolf@uksw.edu.pl
}

Received: 08 January 2014; revised: 18 November 2015; accepted: 19 November 2015; published online: 29 December 2015

\begin{abstract}
In 2009 in this journal it was published the paper of M. K. Singh [1], in which the author presented a six-order variant of Newton's method. Unfortunately, in this paper there were a number of printer errors and a serious error in the proof of theorem on the order of the method proposed. Therefore, we have opted for presenting the correct proof of this theorem.
\end{abstract}

Key words: Newton's method, nonlinear equations, iterative methods, order of convergence

\section{INTRODUCTION}

In [1] the author presented a three-point iterative scheme for solving nonlinear equations of the form

$$
f(x)=0,
$$

where $f: I \subset \mathbb{R} \rightarrow \mathbb{R}$ is a continuously differentiable real function. Starting with a given $x_{0}$ this scheme uses four function evaluations, namely $f\left(x_{n}\right), f^{\prime}\left(x_{n}\right), f^{\prime}\left(y_{n}\right), f\left(z_{n}\right)$ and can be written as

$$
\begin{aligned}
y_{n} & =x_{n}-\frac{f\left(x_{n}\right)}{f^{\prime}\left(x_{n}\right)}, \\
z_{n} & =x_{n}-\frac{f\left(x_{n}\right)\left(f^{\prime}\left(x_{n}\right)+f^{\prime}\left(y_{n}\right)\right)}{f^{\prime}\left(x_{n}\right)^{2}+f^{\prime}\left(y_{n}\right)^{2}}, \\
x_{n+1} & =z_{n}-\frac{f\left(z_{n}\right)\left(f^{\prime}\left(x_{n}\right)^{2}+f^{\prime}\left(y_{n}\right)^{2}\right)}{2 f^{\prime}\left(x_{n}\right) f^{\prime}\left(y_{n}\right)^{2}},
\end{aligned}
$$

where (see Eq. (11) in [1])

$$
\frac{2 f^{\prime}\left(x_{n}\right) f^{\prime}\left(y_{n}\right)^{2}}{f^{\prime}\left(x_{n}\right)^{2}+f^{\prime}\left(y_{n}\right)^{2}} \approx f^{\prime}\left(z_{n}\right)
$$

Apparently this way of replacing $f^{\prime}\left(z_{n}\right)$ by earlier calculated quantities is caused by the intention to reduce the cost of the procedure by decreasing the number of evaluations of $f(\cdot)$ and $f^{\prime}(\cdot)$ to have higher efficiency index (see Def. II.2 in [1]). It should be mentioned that in the original paper the denominator of the last equation (1) contains $f^{\prime}\left(y_{n}\right)$ instead of $f^{\prime}\left(y_{n}\right)^{2}$.

\section{CONVERGENCE ANALYSIS}

The main theorem on the order of the method (1) presented in [1] (Theorem IV.1 on page 187) is as follows:

Let $\alpha \in I$ be a simple zero of a sufficiently differentiable function $f: I \subset \mathbb{R} \rightarrow \mathbb{R}$ for an open interval I. If $x_{0}$ is sufficiently close to $\alpha$, then the method defined by (1) has six-th order convergence.

The proof contains a number of misprints. However, the careful reader can easily fix these errors and guess the correct form of adequate equations. The serious error is at the 
end of proof. Using the approximation (2) the last line of the formula for $x_{n+1}$ can be written in the form (see page 189 in [1])

$$
\begin{gathered}
x_{n+1}=\alpha+\left(2 C_{2}^{2}+\frac{C_{3}}{2}\right) e_{n}^{3} \\
+\mathcal{O}\left(e_{n}^{4}\right)-\frac{\left(2 C_{2}^{2}+\frac{C_{3}}{2}\right) e_{n}^{3}+\mathcal{O}\left(e_{n}^{4}\right)}{1+A e_{n}^{3}+\mathcal{O}\left(e_{n}^{4}\right)} .
\end{gathered}
$$

We use here the symbols $C_{j}$ introduced in the not labeled equation below eq.(13) in the paper of Singh

$$
C_{j}=\frac{1}{j !} \frac{f^{j}(\alpha)}{f^{\prime}(\alpha)} .
$$

From (2) it follows that

$$
\begin{aligned}
& x_{n+1}=\alpha+\left(2 C_{2}^{2}+\frac{C_{3}}{2}\right) e_{n}^{3}+\mathcal{O}\left(e_{n}^{4}\right)-\left(2 C_{2}^{2}+\frac{C_{3}}{2}\right) e_{n}^{3} \\
& -\mathcal{O}\left(e_{n}^{4}\right)=\alpha+\mathcal{O}\left(e_{n}^{4}\right)=\alpha+B e_{n}^{4}+\mathcal{O}\left(e_{n}^{5}\right),
\end{aligned}
$$

where $B$ is some constant. It suggests that the error equation is of the form

However, Singh claims that

$$
e_{n+1}=B e_{n}^{4}+\mathcal{O}\left(e_{n}^{5}\right) .
$$

$$
e_{n+1}=A e_{n}^{6}+\mathcal{O}\left(e_{n}^{7}\right),
$$

what seems to contradict the above short reasoning. Miraculously, it turns out that the form of eq. (24) in [1] does not depend on the terms smaller than $e_{n}^{5}$ because they cancel out in the final expression: the terms $e_{n}^{4}$ and $e_{n}^{5}$ hidden in the Big-Oh symbols $\mathcal{O}\left(e_{n}^{4}\right)$ subtract each other leaving non-zero term $e_{n}^{6}$ and indeed Singh method is of the sixth order, what can be proved in details as follows.

We have

$$
\begin{aligned}
f\left(x_{n}\right) & =f\left(\alpha+e_{n}\right)=f^{\prime}(\alpha) e_{n}+f^{\prime \prime}(\alpha) \frac{e_{n}^{2}}{2 !}+f^{\prime \prime \prime}(\alpha) \frac{e_{n}^{3}}{3 !} \\
& +f^{I V}(\alpha) \frac{e_{n}^{4}}{4 !}+f^{V}(\alpha) \frac{e_{n}^{5}}{5 !}+f^{V I}(\alpha) \frac{e_{n}^{6}}{6 !}+\mathcal{O}\left(e_{n}^{7}\right)= \\
& =f^{\prime}(\alpha)\left[e_{n}+C_{2} e_{n}^{2}+C_{3} e_{n}^{3}+C_{4} e_{n}^{4}\right. \\
& \left.+C_{5} e_{n}^{5}+C_{6} e_{n}^{6}+\mathcal{O}\left(e_{n}^{7}\right)\right] \\
f^{\prime}\left(x_{n}\right) & =f^{\prime}(\alpha)\left[1+2 C_{2} e_{n}+3 C_{3} e_{n}^{2}+4 C_{4} e_{n}^{3}\right. \\
& \left.+5 C_{5} e_{n}^{4}+6 C_{6} e_{n}^{5}+\mathcal{O}\left(e_{n}^{7}\right)\right]
\end{aligned}
$$

From the above equations it follows that

$$
\begin{gathered}
\frac{f\left(x_{n}\right)}{f^{\prime}\left(x_{n}\right)}=\frac{e_{n}+C_{2} e_{n}^{2}+C_{3} e_{n}^{3}+C_{4} e_{n}^{4}}{+C_{5} e_{n}^{5}+C_{6} e_{n}^{6}+\mathcal{O}\left(e_{n}^{7}\right)} \\
\begin{array}{c}
+2 C_{2} e_{n}+3 C_{3} e_{n}^{2}+4 C_{4} e_{n}^{3} \\
+5 C_{5} e_{n}^{4}+6 C_{6} e_{n}^{5}+7 C_{7} e_{n}^{6}+\mathcal{O}\left(e_{n}^{7}\right)
\end{array} \\
=e_{n}-C_{2} e_{n}^{3}-\left(2 C_{3}-2 C_{2}^{2}\right) e_{n}^{3}-B_{4} e_{n}^{4}-B_{5} e_{n}^{5}-B_{6} e_{n}^{6}+\mathcal{O}\left(e_{n}^{7}\right)
\end{gathered}
$$

where $B_{k},(k=4,5,6)$ denote some constants containing $C_{j}$. From (5) and the first equation in (1) we get

$$
\begin{aligned}
y_{n} & =\alpha+C_{2} e_{n}^{2}+\left(2 C_{3}-2 C_{2}^{2}\right) e_{n}^{3}+B_{4} e_{n}^{4} \\
& +B_{5} e_{n}^{5}+B_{6} e_{n}^{6}+\mathcal{O}\left(e_{n}^{7}\right) .
\end{aligned}
$$

Let us denote

$$
\begin{aligned}
g\left(e_{n}\right) & =C_{2} e_{n}^{2}+\left(2 C_{3}-2 C_{2}^{2}\right) e_{n}^{3}+B_{4} e_{n}^{4} \\
& +B_{5} e_{n}^{5}+B_{6} e_{n}^{6}+\mathcal{O}\left(e_{n}^{7}\right) .
\end{aligned}
$$

From (6) we have

$$
g\left(e_{n}\right)^{2}=C_{2}^{2} e_{n}^{4}+D_{5} e_{n}^{5}+D_{6} e_{n}^{6}+\mathcal{O}\left(e_{n}^{7}\right) .
$$

and

$$
g\left(e_{n}\right)^{3}=C_{2}^{3} e_{n}^{6}+\mathcal{O}\left(e_{n}^{7}\right),
$$

where $D_{k},(k=5,6)$ are again some constants containing $C_{j}$. Since

$$
\begin{aligned}
f^{\prime}\left(y_{n}\right) & =f^{\prime}\left(\alpha+g\left(e_{n}\right)\right)=f^{\prime}(\alpha)+f^{\prime \prime}(\alpha) g\left(e_{n}\right) \\
& +f^{\prime \prime \prime}(\alpha) \frac{g\left(e_{n}\right)^{2}}{2 !}+f^{I V}(\alpha) \frac{g\left(e_{n}\right)^{3}}{3 !}+\mathcal{O}\left(e_{n}^{8}\right)
\end{aligned}
$$

then, using (6), (7) and (8), we get

$$
\begin{aligned}
f^{\prime}\left(y_{n}\right) & =f^{\prime}(\alpha)+f^{\prime \prime}(\alpha)\left[C_{2} e_{n}^{2}+\left(2 C_{3}-2 C_{2}^{2}\right) e_{n}^{3}\right. \\
& \left.+B_{4} e_{n}^{4}+B_{5} e_{n}^{5}+B_{6} e_{n}^{6}\right]
\end{aligned}
$$

$+\frac{f^{\prime \prime \prime}(\alpha)}{2 !}\left(C_{2}^{2} e_{n}^{4}+D_{5} e_{n}^{5}+D_{6} e_{n}^{6}\right)+\frac{f^{I V}(\alpha)}{3 !} C_{2}^{3} e_{n}^{6}+\mathcal{O}\left(e_{n}^{7}\right)$.

Using (3) the above equation yields

$$
\begin{aligned}
f^{\prime}\left(y_{n}\right) & =f^{\prime}(\alpha)\left[1+2 C_{2}^{2} e_{n}^{2}+\left(4 C_{2} C_{3}-4 C_{2}^{3}\right) e_{n}^{3}\right. \\
& \left.+E_{4} e_{n}^{4}+E_{5} e_{n}^{5}+E_{6} e_{n}^{6}+\mathcal{O}\left(e_{n}^{7}\right)\right],
\end{aligned}
$$

where $E_{k},(k=4,5,6)$ denote constants containing $C_{j}$.

On the basis of the second equation in (4) we have

$$
\begin{aligned}
& f^{\prime}\left(x_{n}\right)=f^{\prime}(\alpha)^{2}\left[1+4 C_{2}^{2} e_{n}+\left(4 C_{2}^{2}+6 C_{3}\right) e_{n}^{2}\right. \\
& \left.+\left(12 C_{2} C_{3}+8 C_{4}\right) e_{n}^{3}+F_{4} e_{n}^{4}+F_{5} e_{n}^{5}+F_{6} e_{n}^{6}+\mathcal{O}\left(e_{n}^{7}\right)\right],
\end{aligned}
$$

and on the basis of (9)

$$
\begin{aligned}
f^{\prime}\left(y_{n}\right)^{2} & =f^{\prime}(\alpha)^{2}\left[1+4 C_{2}^{2} e_{n}^{2}+\left(8 C_{2} C_{3}-8 C_{2}^{3}\right) e_{n}^{3}\right. \\
& \left.+G_{4} e_{n}^{4}+G_{5} e_{n}^{5}+G_{6} e_{n}^{6}+\mathcal{O}\left(e_{n}^{7}\right)\right],
\end{aligned}
$$

where $F_{k}$ and $G_{k},(k=4,5,6)$ are other constants containing $C_{j}$. The formulas (10) and (11) yield

$$
\begin{aligned}
& f^{\prime}\left(x_{n}\right)^{2}+f^{\prime}\left(y_{n}\right)^{2}=2 f^{\prime}(\alpha)^{2}\left[1+C_{2} e_{n}+\left(4 C_{2}^{2}+3 C_{3}\right) e_{n}^{2}\right. \\
& \left.+\left(10 C_{2} C_{3}+4 C_{4}-4 C_{2}^{3}\right) e_{n}^{3}+H_{4} e_{n}^{4}+H_{5} e_{n}^{5}+H_{6} e_{n}^{6}+\mathcal{O}\left(e_{n}^{7}\right)\right],
\end{aligned}
$$

with some constants $H_{k}(k=4,5,6)$ containing $C_{j}$. Further, from the second equation in (4) and (9) we have

$$
\begin{gathered}
f^{\prime}\left(x_{n}\right)+f^{\prime}\left(y_{n}\right)=2 f^{\prime}(\alpha)\left[1+C_{2} e_{n}+\left(C_{2}^{2}+\frac{3}{2} C_{3}\right) e_{n}^{2}\right. \\
\left.+\left(2 c_{2} C_{3}+2 C_{4}-2 C_{2}^{3}\right) e_{n}^{3}+I_{4} e_{n}^{4}+I_{5} e_{n}^{5}+I_{6} e_{n}^{6}+\mathcal{O}\left(e_{n}^{7}\right)\right],
\end{gathered}
$$
where $I_{k},(k=4,5,6)$ are constants containing $C_{j}$. The above relation and the first formula in (4) yield

$$
\begin{gathered}
f\left(x_{n}\right)\left(f^{\prime}\left(x_{n}\right)+f^{\prime}\left(y_{n}\right)\right)=2 f^{\prime}(\alpha)^{2}\left[e_{n}+C_{2} e_{n}^{2}\right. \\
\left.+\left(2 C_{2}^{2}+\frac{5}{2} C_{3}\right) e_{n}^{3}+J_{4} e_{n}^{4}+J_{5} e_{n}^{5}+J_{6} e_{n}^{6}+\mathcal{O}\left(e_{n}^{7}\right)\right],
\end{gathered}
$$


with constants $J_{k}(k=4,5,6)$ containing $C_{j}$. Now, we can write an expansion for $z_{n}$ given by the second equation in (1). From (12) and (13) we have

$$
\begin{aligned}
& z_{n}=x_{n}-\left[e_{n}+2 C_{2} e_{n}^{2}+\left(2 C_{2}^{2}+\frac{5}{2} C_{3}\right) e_{n}^{3}+J_{4} e_{n}^{4}\right. \\
& \left.+J_{5} e_{n}^{5}+J_{6} e_{n}^{6}+\mathcal{O}\left(e_{n}^{7}\right)\right] /\left[1+2 C_{2} e_{n}+\left(4 C_{2}^{2}+3 C_{3}\right) e_{n}^{2}\right. \\
& +\left(10 C_{2} C_{3}+4 C_{4}-4 C_{2}^{3}\right) e_{n}^{3}+H_{4} e_{n}^{4}+H_{5} e_{n}^{5}+H_{6} e_{n}^{6} \\
& \left.+\mathcal{O}\left(e_{n}^{7}\right)\right]=x_{n}-e_{n}+\left(2 C_{2}^{2}+\frac{1}{2} C_{3}\right) e_{n}^{3}+K_{4} e_{n}^{4}+K_{5} e_{n}^{5} \\
& +K_{6} e_{n}^{6}+\mathcal{O}\left(e_{n}^{7}\right)=\alpha+\left(2 C_{2}^{2}+\frac{1}{2} C_{3}\right) e_{n}^{3}+K_{4} e_{n}^{4}+K_{5} e_{n}^{5} \\
& +K_{6} e_{n}^{6}+\mathcal{O}\left(e_{n}^{7}\right),
\end{aligned}
$$

where $K_{k},(k=4,5,6)$ are other constants containing $C_{j}$. From (14) it follows that

$$
\begin{aligned}
f\left(z_{n}\right) & =f\left(\alpha+\left(2 C_{2}^{2}+\frac{1}{2} C_{3}\right) e_{n}^{3}+K_{4} e_{n}^{4}\right. \\
& \left.+K_{5} e_{n}^{5}+K_{6} e_{n}^{6}+\mathcal{O}\left(e_{n}^{7}\right)\right) .
\end{aligned}
$$

Let us denote

$$
\begin{aligned}
h\left(e_{n}\right) & =\left(2 C_{2}^{2}+\frac{1}{2} C_{3}\right) e_{n}^{3}+K_{4} e_{n}^{4} \\
& +K_{5} e_{n}^{5}+K_{6} e_{n}^{6}+\mathcal{O}\left(e_{n}^{7}\right) .
\end{aligned}
$$

Then we can write

$$
\begin{gathered}
f\left(z_{n}\right)=f\left(\alpha+h\left(e_{n}\right)\right)=f^{\prime}(\alpha) h\left(e_{n}\right)+f^{\prime \prime}(\alpha) \frac{h\left(e_{n}\right)^{2}}{2 !} \\
+\mathcal{O}\left(h\left(e_{n}\right)^{3}\right)=f^{\prime}(\alpha) h\left(e_{n}\right)+f^{\prime \prime}(\alpha) \frac{h\left(e_{n}\right)^{2}}{2 !}+\mathcal{O}\left(e_{n}^{9}\right) .
\end{gathered}
$$

because in (15) the term $e_{n}$ occurs at least in the third power. Since from (15) it also follows that

$$
h\left(e_{n}\right)^{2}=\left(2 C_{2}^{2}+\frac{1}{2} C_{3}\right)^{2} e_{n}^{6}+\mathcal{O}\left(e_{n}^{7}\right),
$$

then from (16) we get

$$
\begin{gathered}
f\left(z_{n}\right)=f^{\prime}(\alpha)\left[\left(2 C_{2}^{2}+\frac{1}{2} C_{3}\right) e_{n}^{3}+K_{4} e_{n}^{4}+K_{5} e_{n}^{5}\right. \\
\left.+K_{6} e_{n}^{6}+\mathcal{O}\left(e_{n}^{7}\right)\right]+C_{2} f^{\prime}(\alpha)\left[\left(2 C_{2}^{2}+\frac{1}{2} C_{3}\right)^{2} e_{n}^{6}\right. \\
\left.+\mathcal{O}\left(e_{n}^{7}\right)\right]+\mathcal{O}\left(e_{n}^{9}\right)=f^{\prime}(\alpha)\left[\left(2 C_{2}^{2}+\frac{1}{2} C_{3}\right) e_{n}^{3}+K_{4} e_{n}^{4}\right. \\
\left.+K_{5} e_{n}^{5}+\left(K_{6}+C_{2}\left(2 C_{2}^{2}+\frac{1}{2} C_{3}\right)^{2}\right) e_{n}^{6}+\mathcal{O}\left(e_{n}^{7}\right)\right]
\end{gathered}
$$

Inserting the second relation in (4), (11), (12), (14) and (17) into the last relation in (1) we have

$$
\begin{aligned}
x_{n+1} & =\alpha+\left(2 C_{2}^{2}+\frac{1}{2} C_{3}\right) e_{n}^{3}+K_{4} e_{n}^{4}+K_{5} e_{n}^{5}+K_{6} e_{n}^{6} \\
& +\mathcal{O}\left(e_{n}^{7}\right)-\left\{f ^ { \prime } ( \alpha ) \left[\left(2 C_{2}^{2}+\frac{1}{2} C_{3}\right) e_{n}^{3}+K_{4} e_{n}^{4}+K_{5} e_{n}^{5}\right.\right. \\
& \left.+\left(K_{6}+C_{2}\left(2 C_{2}^{2}+\frac{1}{2} C_{3}\right)^{2}\right) e_{n}^{6}+\mathcal{O}\left(e_{n}^{7}\right)\right] \times 2 f^{\prime}(\alpha)^{2} \\
& \times\left[1+2 C_{2} e_{n}+\left(4 C_{2}^{2}+3 C_{3}\right) e_{n}^{2}+\left(10 C_{2} C_{3}\right.\right. \\
& \left.+4 C_{4}-4 C_{2}^{3}\right) e_{n}^{3}+H_{4} e_{n}^{4}+H_{5} e_{n}^{5}+H_{6} e_{n}^{6} \\
& \left.\left.+\mathcal{O}\left(e_{n}^{7}\right)\right]\right\} /\left\{2 f ^ { \prime } ( \alpha ) \left[1+2 C_{2} e_{n}+3 C_{3} e_{n}^{2}+4 C_{4} e_{n}^{3}\right.\right. \\
& \left.+5 C_{5} e_{n}^{4}+6 C_{6} e_{n}^{5}+7 C_{7} e_{n}^{6}+\mathcal{O}\left(e_{n}^{7}\right)\right] \\
& \times f^{\prime}(\alpha)^{2}\left[1+4 C_{2}^{2} e_{n}^{2}+\left(8 C_{2} C_{3}-8 C_{2}^{3}\right) e_{n}^{3}\right. \\
& \left.\left.+G_{4} e_{n}^{4}+G_{5} e_{n}^{5}+G_{6} e_{n}^{6}+\mathcal{O}\left(e_{n}^{7}\right)\right]\right\}= \\
& =\alpha+\left(2 C_{2}^{2}+\frac{1}{2} C_{3}\right) e_{n}^{3}+K_{4} e_{n}^{4}+K_{5} e_{n}^{5}+K_{6} e_{n}^{6} \\
& +\mathcal{O}\left(e_{n}^{7}\right)-\left[\left(2 C_{2}^{2}+\frac{1}{2} C_{3}\right) e_{n}^{3}+L_{5} e_{n}^{5}+L_{6} e_{n}^{6}\right. \\
& \left.+\mathcal{O}\left(e_{n}^{7}\right)\right] /\left[1+C_{2} e_{n}+\left(4 C_{2}^{2}+3 C_{3}\right) e_{n}^{2}\right. \\
& +\left(5 C_{2}^{3}+8 C_{2} C_{3}+4 C_{4}\right) e_{n}^{3}+M_{4} e_{n}^{4} \\
& \left.+M_{5} e_{n}^{5}+M_{6} e_{n}^{6}+\mathcal{O}\left(e_{n}^{7}\right)\right]
\end{aligned}
$$

where $L_{k}$ and $M_{k},(k=4,5,6)$ are consecutive constants containing some combinations of $C_{j}$. In particular, we have

$$
\begin{aligned}
L_{4} & =K_{4}+2 C_{2}\left(2 C_{2}^{2}+\frac{1}{2} C_{3}\right), \\
L_{5} & =K_{5}+2 C_{2} K_{4}+\left(2 C_{2}^{2}+\frac{1}{2} C_{3}\right)\left(4 C_{2}^{2}+3 C_{3}\right), \\
L_{6} & =K_{6}+2 C_{2} K_{5}+\left(4 C_{2}^{2}+3 C_{3}\right) K_{4}+\left(2 C_{2}^{2}+\frac{1}{2} C_{3}\right) \\
& \times\left(\frac{21}{2} C_{2} C_{3}+4 C_{4}-2 C_{2}^{3}\right)
\end{aligned}
$$

(the forms of $M_{k}$ are unimportant further). If we denote

$$
\begin{array}{r}
K_{3}=L_{3}=2 C_{2}^{2}+\frac{1}{2} C_{3}, \\
M_{1}=2 C_{2}, \\
M_{2}=4 C_{2}^{2}+3 C_{3}, \\
M_{3}=4 C_{2}^{3}+8 C_{2} C_{3}+4 C_{4},
\end{array}
$$

then the equation (18) can be written in a simpler form

$$
\begin{gathered}
x_{n+1}=\alpha+K_{3} e_{n}^{3}+K_{4} e_{n}^{4}+K_{5} e_{n}^{5}+K_{6} e_{n}^{6}+\mathcal{O}\left(e_{n}^{7}\right) \\
-\frac{L_{3} e_{n}^{3}+L_{4} e_{n}^{4}+L_{5} e_{n}^{5}+L_{6} e_{n}^{6}+\mathcal{O}\left(e_{n}^{7}\right)}{1+M_{1} e_{n}+M_{2} e_{n}^{2}+M_{3} e_{n}^{3}+M_{4} e_{n}^{4}} \\
+M_{5} e_{n}^{5}+M_{6} e_{n}^{6}+\mathcal{O}\left(e_{n}^{7}\right)
\end{gathered}
$$


Denoting the fraction in (21) by $\Phi$ it can be calculated that

$$
\begin{aligned}
& \Phi=L_{3} e_{n}^{3}+\left(L_{4}-M_{1} L_{3}\right) e_{n}^{4}+\left[L_{5}-M_{1} L_{4}\right. \\
+ & \left.\left(M_{1}^{2}-M_{2}\right) L_{3}\right] e_{n}^{5}+\left[L_{6}-M_{1} L_{5}+\left(M_{1}^{2}-M_{2}\right) L_{4}\right. \\
+ & \left.\left(2 M_{2} M_{2}-M_{1}^{3}-M_{3}\right) L_{3}\right] e_{n}^{6}+\mathcal{O}\left(e_{n}^{7}\right) .
\end{aligned}
$$

But from (19) and (20) it follows that

$$
\begin{aligned}
L_{3} & =K_{3}, \\
L_{4} & =K_{4}+M_{1} L_{3}, \\
L_{5} & =K_{5}+M_{1} K_{4}+M_{2} L_{3}, \\
L_{6} & =K_{6}+M_{1} K_{5}+M_{2} K_{4} \\
& +\left(\frac{21}{2} C_{2} C_{3}+4 C_{4}-2 C_{2}^{3}\right) L_{3} .
\end{aligned}
$$

Inserting these results in (22) we obtain

$$
\begin{aligned}
& \Phi=K_{3} e_{n}^{3}+K_{4} e_{n}^{4}+K_{5} e_{n}^{5} \\
& +\left[K_{6}+\left(\frac{5}{2} C_{2} C_{3}-6 C_{2}^{3}\right) K_{3}\right] e_{n}^{6}+\mathcal{O}\left(e_{n}^{7}\right) .
\end{aligned}
$$

Thus, from (21) we finally have

$$
x_{n+1}=\alpha+C_{2}\left(6 C_{2}^{2}-\frac{5}{2} C_{3}\right) e_{n}^{6}+\mathcal{O}\left(e_{n}^{7}\right) .
$$

The last relation states that the Singh method is of the sixth order and now the proof is correct. On page 186 in [1]
Singh defines efficiency index as $p^{1 / m}$, where $p$ is the order of the method and $m$ is the number of functions evaluations required by the method. Usual Newton's method is of order 2 and requires 2 functions evaluations, therefore the efficiency index of Newton's method is $2^{1 / 2}=1.414 \ldots$. The whole algorithm of Singh presented in eq. (1) contains 4 function evaluations, 5 multiplication and 3 divisions, while the usual Newton method three times iterated has the order eight with 6 function evaluations and 3 divisions without any multiplication involved. Thus three times iterated Newton's method has the efficiency index $8^{1 / 6}=\sqrt{2}=1.4142$ (the same as usual Newton method) in contrast to the efficiency index of the Singh's method $6^{1 / 4}=1.5651$. However the advantage of the method proposed by Singh seems to us to be illusive in view of complicated character of computer implementation and time consuming extra operations (intermediate results of arithmetical operations have to be stored in temporary variables).

\section{References}

[1] M. K. Singh, A Six-order Variant of Newton's Method for Solving Nonlinear Equations, Computational Methods in Science and Technology 15(2), 185-193 (2009).

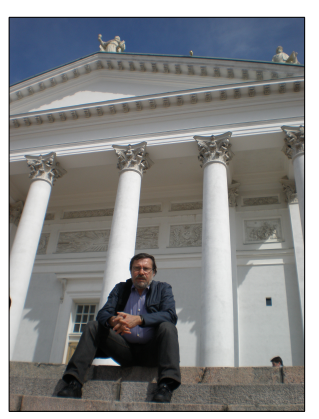

Andrzej Marciniak was born in Poznań (Poland) in 1953. He received the MSc degree in mathematics in 1977, the MSc degree in astronomy in 1979 and PhD degree in mathematics in 1981, all from the Adam Mickiewicz University in Poznań. In 1993 he received the Dr. Habil. degree in physics from the Nicolaus Copernicus University in Torun (Poland) and in 2010 he received the Professor Title from the President of Poland. From 1977 to 1987 and from 2000 to 2011 he held a research position at the Faculty of Mathematics and Computer Science of the Adam Mickiewicz University, and since 1987 he has been an assistant professor in Institute of Mathematics and then a professor of computer science at the Faculty of Computing Science of the Poznań University of Technology. From 2005 to 2007 he was a member of the Polish Accreditation Committee, an expert of which he has been up to this day. From 2005 to 2008 he held the office of the President of Polish Information Processing Society. Since 2013 he has also been working at the Higher Vocational State School of President Stanisław Wojciechowski in Kalisz (Poland), where he has been managing the Department of Computer Science. His research interests include computer programming and numerical methods, especially for solving ordinary and partial differential equations with applications to dynamical problems. In these fields he wrote three monographs, more than 20 textbooks and a number of scientific articles. During his work he has promoted three $\mathrm{PhD}$ dissertations in informatics.

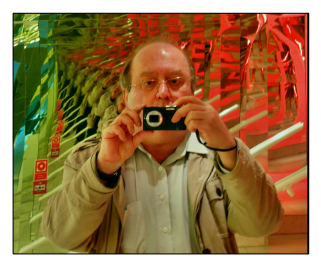

Marek Wolf obtained PhD degree in physics (1982) and habilitation (1993) at the Wroclaw University, where he was employed till 2011. Since 1984 he has been doing computer experiments in physics and mathematics. In 1991 and 1993 he was a research fellow at the Center for Polymer Studies at the Boston University. Since 2011 he is a member of the Faculty of Mathematics and Natural Sciences, College of Sciences at the Cardinal Stefan Wyszynski University in Warsaw. His hobby is photography. 